\title{
Philosophiques
}

\section{Droits humains et minorités culturelles}

\section{Amandine Catala}

Volume 42, numéro 2, automne 2015

Dossier. Les droits humains - nouveaux développements

URI : https://id.erudit.org/iderudit/1034740ar

DOI : https://doi.org/10.7202/1034740ar

Aller au sommaire du numéro

\section{Éditeur(s)}

Société de philosophie du Québec

ISSN

0316-2923 (imprimé)

1492-1391 (numérique)

Découvrir la revue

Citer cet article

Catala, A. (2015). Droits humains et minorités culturelles. Philosophiques, 42(2), 231-250. https://doi.org/10.7202/1034740ar

\section{Résumé de l'article}

J'aborde tout d'abord l'objection relativiste aux droits humains, afin de pouvoir ensuite me concentrer sur d'autres questions soulevées par la question des droits humains et des minorités culturelles. Le but de ma discussion est d'identifier et d'interroger les tensions potentielles entre minorités culturelles et droits humains, afin de montrer en quoi les droits humains peuvent protéger les minorités culturelles et, ultimement, de problématiser la manière dont cette protection peut se déployer. Dans ce but, je commence par clarifier deux notions-clés de ce débat : les minorités culturelles et les droits différenciés selon l'appartenance à un groupe, en en proposant une typologie (section 1). J'examine ensuite dans quelle mesure les aspects collectiviste et particulariste des droits différenciés se trouvent ou non en tension respectivement avec les aspects individualiste et universaliste des droits humains (section 2). Prenant comme points de référence quelques droits humains particuliers, je me penche enfin sur la situation de certaines minorités culturelles dans les démocraties libérales occidentales pour dégager les conditions délibératives de la réalisation de ces droits humains (section 3). $\mathrm{Au}$ fil de ces trois sections, une de mes préoccupations est de mettre en lumière les enjeux méthodologiques qui sous-tendent ces différents débats et de montrer la nécessité d'adopter une approche améliorative.
Tous droits réservés (C) Société de philosophie du Québec, 2015
Ce document est protégé par la loi sur le droit d'auteur. L’utilisation des services d'Érudit (y compris la reproduction) est assujettie à sa politique d'utilisation que vous pouvez consulter en ligne.

https://apropos.erudit.org/fr/usagers/politique-dutilisation/ 


\title{
Droits humains et minorités culturelles
}

\author{
AMANDINE CATALA \\ Université du Québec à Montréal \\ catala.amandine@uqam.ca
}

\begin{abstract}
RÉSUMÉ. - J'aborde tout d'abord l'objection relativiste aux droits humains, afin de pouvoir ensuite me concentrer sur d'autres questions soulevées par la question des droits humains et des minorités culturelles. Le but de ma discussion est d'identifier et d'interroger les tensions potentielles entre minorités culturelles et droits humains, afin de montrer en quoi les droits humains peuvent protéger les minorités culturelles et, ultimement, de problématiser la manière dont cette protection peut se déployer. Dans ce but, je commence par clarifier deux notions-clés de ce débat: les minorités culturelles et les droits différenciés selon l'appartenance à un groupe, en en proposant une typologie (section 1). J'examine ensuite dans quelle mesure les aspects collectiviste et particulariste des droits différenciés se trouvent ou non en tension respectivement avec les aspects individualiste et universaliste des droits humains (section 2). Prenant comme points de référence quelques droits humains particuliers, je me penche enfin sur la situation de certaines minorités culturelles dans les démocraties libérales occidentales pour dégager les conditions délibératives de la réalisation de ces droits humains (section 3). Au fil de ces trois sections, une de mes préoccupations est de mettre en lumière les enjeux méthodologiques qui sous-tendent ces différents débats et de montrer la nécessité d'adopter une approche améliorative.
\end{abstract}

ABSTRACT. - I first address the relativist challenge to human rights, so as to be able to focus next on other questions raised by the question of human rights and cultural minorities. The aim of my discussion is to identify and to question the potential tensions between cultural minorities and human rights, in order to show how human rights can protect cultural minorities, and ultimately to problematize the way in which this protection can take place. To that end, I start by clarifying two key notions in this debate: cultural minorities and group-differentiated rights, by proposing a typology (section 1 ). I then examine to what extent the collectivist and the particularist aspects of group-differentiated rights are in tension respectively with the individualist and the universalist aspects of human rights. Taking as reference points a few particular human rights, I turn, finally, to the situation of certain cultural minorities in western liberal democracies to draw out the deliberative conditions of the realization of those human rights (section 3). Throughout all three sections, one of my concerns is to shed light on the methodological stakes underlying those different debates and to show that we should adopt an ameliorative approach.

\section{Introduction: S'accorder au sujet des droits humains}

Le sujet des droits humains et des minorités culturelles est un vaste sujet, soulevant de nombreuses questions et pouvant donc être abordé sous les différents angles que constituent ces différentes questions. Une de ces questions, qui a fait couler beaucoup d'encre, est l'objection aux droits humains 
que l'on pourrait appeler le défi relativiste. Si cette objection s'avère être un défi digne de ce nom, elle pourrait rendre futile toute autre discussion du sujet. Bien que cette objection soit à ce stade largement considérée comme obsolète (Nickel 20I4), je l'aborde malgré tout brièvement d'entrée de jeu afin de pouvoir l'écarter comme erronée et ainsi faire place dans la suite de la discussion à d'autres questions que soulève le débat des droits humains et des minorités culturelles.

Selon l'objection du défi relativiste, les droits humains ne correspondraient pas à la cristallisation de valeurs universellement partagées, mais plutôt à une imposition indue, au reste du monde, de valeurs qui en réalité ne sont propres qu'à l'Occident (Ake I987; Bauer et Bell I999; Talbott 2005). Les droits humains procéderaient ainsi d'une démarche ethnocentrique, voire impérialiste, et seraient donc peu susceptibles d'être reconnus et adoptés par certains pays, certaines cultures, ou certaines minorités culturelles. Autrement dit, la question des droits humains et des minorités culturelles n'en serait pas véritablement une: elle serait un non-problème puisque les uns n'auraient tout simplement rien à voir avec les autres, les droits humains ne concernant pas les minorités culturelles.

En réponse, il convient tout d'abord de remarquer que cette objection se méprend quant à l'objectif de la doctrine des droits humains. Celle-ci vise en effet non à décrire le statu quo moral, mais à réformer le statu quo légal et politique en prescrivant certains buts à atteindre sur ces différents plans. Il ne faut donc pas confondre les droits humains comme normes ou principes visant à guider les actions des États (ou des individus, ou de certaines institutions nationales, infranationales, ou internationales), d'une part, et les différentes justifications pouvant soutenir ces normes de conduite, d'autre part. En d'autres termes, il s'agit de distinguer entre deux niveaux différents: d'une part, le niveau pratique, prescriptif ou normatif; d'autre part, le niveau théorique, fondationnel, que celui-ci s'entende en un sens axiologique (quelles sont les valeurs morales particulières qui sous-tendent les droits humains: individualistes, collectivistes, etc.) ou en un sens métaéthique (quel est le statut ontologique ou la nature ultime des droits humains: reviennent-ils uniquement à des outils légaux ou politiques utiles? correspondent-ils à des droits naturels ou pré-politiques ? etc.). Il est donc possible de s'accorder sur les principes ou objectifs que représentent les droits humains, même si l'on adopte par ailleurs des conceptions différentes, voire incompatibles, en ce qui concerne leurs fondements axiologiques ou métaéthiques (An-Na'im 2003; Beitz 2009; Nickel et Reidy 2008; Taylor 1999).

En outre, l'objection relativiste s'affaiblit davantage dès lors que l'on constate que chaque État membre des Nations Unies a ratifié au moins un des six traités principaux des droits humains ${ }^{1}$; que $80 \%$ en ont ratifié au

1. Ces six traités sont: le Pacte international relatif aux droits civils et politiques; le Pacte international relatif aux droits économiques, sociaux et culturels; la Convention interna- 
moins quatre; que de nombreux pays d'Afrique, des Amériques et d'Europe font partie de régimes régionaux de promotion et de protection des droits humains dotés d'institutions et de cours propres ${ }^{2}$; et que des sondages d'opinion indiquent que l'ouverture et le soutien de la population mondiale envers les droits humains et les institutions les promouvant vont croissant (Bayefsky 200I; Nickel 20I4). Ainsi, la majorité de la population sondée dans des pays aussi divers que l'Argentine, l'Ukraine, l'Azerbaïdjan, l'Égypte, l'Iran, le Kenya, le Nigeria, la Chine, l'Inde et l'Indonésie, a répondu par l'affirmative pour son approbation de droits humains fondamentaux tels que la liberté d'association, la liberté de conscience, l'égalité hommesfemmes et la non-discrimination raciale, ainsi que pour son approbation de la promotion par les Nations Unies des droits humains énoncés dans la Déclaration universelle des droits de l'homme (Nickel 20I4). Ces données empiriques, qui concernent aussi bien les États que les institutions internationales ou les citoyens de par le monde, viennent ainsi démentir l'ampleur du désaccord étant censé régner entre cultures quant aux droits humains. Par ailleurs, quand bien même il y aurait désaccord, souligner un désaccord de fait ne suffirait pas pour autant à invalider la doctrine des droits humains : l'absence d'une réponse commune à une certaine question ne prouve pas pour autant l'absence d'une réponse correcte à cette même question. L'objection relativiste semble donc reposer sur des prémisses empiriques et une logique qui se révèlent douteuses.

Tout cela vient appuyer la position selon laquelle on peut au moins s'accorder minimalement au sujet des droits humains. Cela permet d'écarter l'objection relativiste et de se concentrer à présent sur d'autres questions soulevées par notre sujet des droits humains et des minorités culturelles. Le but de la discussion qui suit sera d'identifier et d'interroger les tensions potentielles entre minorités culturelles et droits humains, afin de montrer en quoi les droits humains peuvent protéger les minorités culturelles; et ultimement, de problématiser la manière dont cette protection peut se déployer. Dans ce but, il sera utile de commencer par clarifier deux notions-clés de ce débat, à savoir ce que l'on entend par les notions de minorités culturelles et de droits différenciés selon l'appartenance à un groupe (group-differentiated rights), en en proposant une typologie (section I). Il sera ensuite important de voir dans quelle mesure les aspects collectiviste et particulariste des droits différenciés se trouvent ou non en tension respectivement avec les aspects individualiste et universaliste des droits humains (section 2). En prenant

tionale sur l'élimination de toutes les formes de discrimination raciale; la Convention internationale sur l'élimination de toutes les formes de discrimination à l'égard des femmes; la Convention internationale des droits de l'enfant; la Convention contre la torture et autres peines ou traitements cruels, inhumains ou dégradants.

2. Par exemple: la Commission et la Cour africaines des droits de l'homme et des peuples; la Commission et la Cour interaméricaines des droits de l'homme; la Cour européenne des droits de l'homme. 
comme points de référence quelques droits humains particuliers, il sera enfin pertinent de se pencher sur la situation de certaines minorités culturelles dans les démocraties libérales occidentales pour dégager les conditions délibératives de la réalisation de ces droits humains, en particulier le droit des minorités culturelles de participer aux décisions nationales ou régionales les concernant (section 3). Au fil de ces trois sections, une de mes préoccupations sera de mettre en lumière les enjeux méthodologiques qui sous-tendent ces différents débats et de montrer la nécessité d'adopter une approche améliorative.

\section{Minorités culturelles et droits différenciés: conceptualisation et typologie}

\subsection{Minorités culturelles}

L'expression de «minorités culturelles» est utilisée pour désigner au moins trois types distincts de groupe minoritaire: les minorités nationales; les minorités ethniques; et les minorités sociales. Une minorité nationale est un groupe territorialement concentré qui (le plus souvent) était autonome (se gouvernait lui-même) avant d'être incorporé au sein de l'État dont il fait actuellement partie, et qui vit en parallèle du groupe national dominant (Kymlicka I995). Les peuples autochtones des Amériques, d'Australie, de Nouvelle-Zélande, ou de Scandinavie; le Québec au Canada, la Catalogne ou le Pays basque en Espagne, ou l'Écosse au Royaume-Uni, sont tous des exemples de minorités nationales. Une minorité ethnique est un groupe qui résulte de l'immigration d'individus ou de familles, le plus souvent suivant des liens historiques coloniaux ou militaires, ou des programmes de travailleurs invités (Higgins 20I3), et qui, à la différence des minorités nationales, vit intégré à la société d'accueil (Kymlicka I995), dans une mesure plus ou moins grande. Les populations d'origine algérienne en France; turque en Allemagne; congolaise ou marocaine en Belgique; portoricaine aux ÉtatsUnis; ou indienne et pakistanaise au Royaume-Uni sont toutes des exemples de minorités ethniques. Une minorité sociale est un groupe qui est le produit de certaines relations de pouvoir à l'œuvre au sein d'une société, qui le placent en position subordonnée par rapport au groupe social dominant issu de ces mêmes relations de pouvoir (Young I990). Ces relations de pouvoir sociales constituent le phénomène spécifique de l'oppression et se traduisent par un ensemble de contraintes formelles et informelles qui désavantage systémiquement et ainsi subordonne les membres de minorités sociales (Frye I983), et qui inversement forme le privilège des groupes dominants (Bailey I998). L'oppression est donc un phénomène structurel qui affecte les individus en tant qu'ils appartiennent à certains groupes sociaux (Bailey I998, Frye 1983, Young I990). Les groupes sociaux définis selon le genre, la race, la classe, l'orientation sexuelle ou les capacités physiques, tels que les femmes, les gens de couleur, les pauvres, les personnes homosexuelles ou les individus handicapés, sont tous des exemples de minorités sociales. 
Chacun des trois types de minorité présentés ici — national, ethnique et social - a donc une genèse propre relevant de processus historiques ou socio-politiques particuliers, qui justifie donc qu'une attention particulière lui soit portée. Les trois types de minorité peuvent être qualifiés de "culturels » en ce que leurs membres partagent sur un certain plan (national, ethnique ou social) un certain mode de vie ou une certaine expérience sociale, c'est-à-dire une certaine culture, entendue dans un sens large. Les cultures nationale, ethnique et sociale se traduisent en effet chacune par un ensemble de croyances, pratiques et institutions formelles et informelles qui forment et informent les opportunités qui s'offrent à leurs membres, et ont donc un impact profond sur leur vie et leur mode d'existence. En raison de leur statut de minorité et donc de leur position de vulnérabilité, ces trois types de minorité requièrent des protections particulières, revendiquées et/ou instituées sous la forme de droits différenciés selon l'appartenance à un groupe (ciaprès simplement «droits différenciés »).

\subsection{Remarque méthodologique}

Avant de passer à la question des droits différenciés, il convient de faire une pause méthodologique en s'arrêtant un instant pour souligner l'importance de reconnaître le troisième type de minorité (les minorités sociales) comme type distinct de minorité justifiant un traitement propre au même titre que les deux premiers (les minorités nationales et ethniques). Il est d'autant plus pertinent de s'arrêter sur ce point que Kymlicka, dont le travail novateur est incontournable dans ces débats, remarque que parvenir à une démocratie plus inclusive et tolérante requiert de réaliser la justice sur les trois fronts. En effet, Kymlicka souligne l'importance de complémenter une approche (comme la sienne) qui traite principalement des droits des minorités nationales et ethniques, par une approche qui se préoccupe des minorités sociales, qui se retrouvent à travers ces différents groupes (I995, I9). Cependant, Kymlicka n'approfondit ni les raisons de cette affirmation ni la manière dont elle pourrait être réalisée. Étant donné les enjeux conceptuels et normatifs soulevés ici, il est important de préciser pourquoi une approche multilatérale est nécessaire, et comment elle pourrait être réalisée.

Sans une approche multilatérale surgirait en effet un risque d'instaurer une hiérarchie dans les priorités qui nuirait à la réalisation de la justice sur de multiples fronts simultanément. Une approche qui subsumerait les préoccupations sociales sous les préoccupations nationales et ethniques reviendrait à prioriser ces dernières au détriment des premières. En effet, valoriser la culture nationale ou ethnique minoritaire semble d'une part nécessaire à la protéger contre ce qui serait sinon une domination par défaut par la culture nationale ou ethnique majoritaire. En même temps, la culture nationale ou ethnique abrite souvent elle-même des injustices pour les minorités sociales qui en font partie. La culture nationale ou ethnique revêt ainsi un statut ambigu par rapport au but global qu'est la réalisation de la justice 
simultanément sur les fronts national ou ethnique ainsi que social, puisqu'elle se révèle être à la fois un lieu de résistance (sur le plan national ou ethnique) et un lieu d'oppression (sur le plan social). Valoriser d'abord et avant tout la culture nationale ou ethnique créerait alors une dichotomie (entre justice nationale ou ethnique, d'une part, et justice sociale, d'autre part) qui tendrait ultimement à nuire aux efforts de justice pour ses minorités sociales.

Il semble dès lors nécessaire de reconnaître les minorités sociales comme type distinct de minorité aux côtés des minorités nationales et ethniques, de manière à pouvoir faire face aux multiples types d'injustice en jeu, sans que les efforts de solutionner les unes viennent nuire aux efforts de solutionner les autres en les renforçant. Rendre visibles simultanément ces différents types de minorité ou d'axe d'appartenance permet de mieux voir la manière dont ils se croisent - et par conséquent, de mieux envisager en quoi la situation des individus situés à leur croisement exige de traiter des deux simultanément, sans les hiérarchiser. Autrement dit, il convient de tirer ici les leçons offertes par la théorie de l'intersectionnalité (Crenshaw I99I). Celle-ci montre en quoi il serait illusoire de prétendre résoudre, par exemple, le problème du patriarcat si les seules expériences prises en considération pour ce faire sont celles des femmes blanches (ce qui renforce le problème du racisme); et en quoi il serait illusoire de prétendre résoudre le problème du racisme si les seules expériences prises en considération pour ce faire sont celles des hommes noirs (ce qui renforce le problème du patriarcat). Cela veut dire que réaliser la justice pour les femmes, les noirs, et les femmes noires exige de tenir compte simultanément des axes du genre et de la race, et de ne pas subordonner l'un à l'autre, sans quoi leurs problèmes respectifs se renforceront mutuellement. De même, il serait illusoire de prétendre résoudre les problèmes des minorités sociales s'ils sont subordonnés à un autre axe d'appartenance (la culture nationale ou ethnique) qui en fait les renforce. Tout comme, inversement, il serait illusoire de prétendre résoudre les problèmes des minorités nationales ou ethniques s'ils sont subordonnés à un autre axe d'appartenance (un groupe social minoritaire). Ainsi, pour traiter de la situation des femmes autochtones, par exemple, il faut tenir compte à la fois de leur appartenance au groupe autochtone (à la différence des femmes non autochtones) et de leur appartenance au groupe femme (à la différence des hommes autochtones). On ne peut donc pas se contenter de subsumer leurs intérêts sous ceux de leur groupe national. Il faut dès lors adopter une approche multilatérale, qui seule peut répondre aux enjeux conceptuels et normatifs soulevés ici.

Cette prescription a une implication méthodologique importante: réaliser nos objectifs de moralité politique (ici, éliminer l'injustice sur les plans national, ethnique et social) requiert d'adopter les concepts et catégories d'analyse qui nous le permettront, c'est-à-dire de saisir l'ontologie sociale pertinente (Haslanger 2000; Haslanger 2005; Mills 2005). Cela requiert de s'interroger non seulement sur qui sont les agents pertinents (ici, les diffé- 
rents types de minorité culturelle), mais également sur la manière dont leurs interrelations peuvent être correctement saisies (ici, l'intersection de ces multiples axes d'appartenance). Afin de ne pas se fourvoyer dans la partie normative d'un projet - avant de pouvoir théoriser les droits et les devoirs qui s'imposent - il faut au préalable ne pas se tromper dans sa partie descriptive: si le but est de transformer une situation injuste en une situation juste, il nous faut auparavant comprendre les dynamiques qui génèrent et maintiennent cette situation d'injustice (Mills 2005). Les analyses qu'offrent respectivement Young de l'oppression et Crenshaw de l'intersectionnalité (tout comme l'analyse de la culture que j'offrirai dans la troisième partie de cet article) remplissent cette exigence méthodologique: si on peut identifier et comprendre les mécanismes qui génèrent et maintiennent certaines structures de domination, on peut commencer à voir la manière dont il convient de réformer ces structures.

Autrement dit, lorsqu'il est question de formuler des concepts ou des catégories d'analyse, il convient d'adopter une approche améliorative, par contraste avec une approche purement conceptuelle ou purement descriptive (Haslanger 2000; Haslanger 2005). L'approche conceptuelle part de la question épistémologique "Quel est notre concept de X: quelles sont ses caractéristiques définitionnelles?» et cherche à y répondre en suivant une réflexion intuitive ou a priori, sans en interroger l'utilité théorique ou pratique. L'approche descriptive procède quant à elle à une enquête a posteriori afin de répondre à la question ontologique "Quelles sont les choses qui sont saisies par X: quels sont les objets qui présentent ses caractéristiques définitionnelles? » L'approche améliorative, pour sa part, ne cherche pas simplement à expliquer la compréhension ordinaire d'un certain concept (comme l'approche conceptuelle), ni simplement à identifier son extension empirique (comme l'approche descriptive). L'approche améliorative part plutôt de la question politique «À quoi le concept X sert-il sur le plan théorique et pratique: est-il utile pour atteindre nos buts légitimes?». Par exemple, si l'on adopte l'approche conceptuelle et que l'on définit le concept femme dans le sens ordinaire, seulement selon certaines caractéristiques physiques ou biologiques, le concept ne nous sera pas véritablement utile pour expliquer et réformer les inégalités qui caractérisent la situation des femmes; pas plus qu'il ne nous sera utile à cet égard d'adopter l'approche descriptive et d'identifier les différents individus qui présentent ces caractéristiques physiques ou biologiques. Par contre, si l'on adopte l'approche améliorative et que l'on définit le concept femme avant tout comme une catégorie politique résultant de certaines relations de pouvoir sociales (catégorie elle-même toujours traversée par d'autres catégories politiques comme la race, la classe ou l'orientation sexuelle), on se trouve alors en possession d'un concept politiquement utile. Comme l'illustre l'exemple du concept femme, l'approche améliorative détermine l'extension du concept (ce à quoi le concept réfère empiriquement). Elle permet donc d'identifier l'ontologie 
pertinente (les objets pertinents et éventuellement les relations de pouvoir dont ils résultent). On est alors mieux équipé pour commencer à saisir et à défaire ces relations de domination et de subordination. Cela permet de parvenir au but politique ou critique fixé comme objectif au départ.

Il y a donc bien un enjeu conceptuel (épistémologique) et descriptif (ontologique) dans la démarche améliorative (politique), mais l'ordre dans la manière de procéder est inversé: on s'interroge d'abord sur nos buts politiques, et ensuite on peut définir un concept et saisir son extension. Autrement dit, notre ontologie ou notre description du monde à l'aide de certains concepts et catégories d'analyse découle de nos buts critiques, et non l'inverse: «le monde à lui seul ne peut nous dire [ce à quoi correspondent nos concepts]; c'est à nous de décider ce à quoi [...] ils correspondent dans le monde» (Haslanger 2000, 34).

Pourquoi favoriser l'approche améliorative? Parce que notre but est de rendre compte de certaines injustices pour pouvoir identifier des solutions y remédiant. Pour atteindre ce but, nous avons besoin d'outils efficaces, qui nous permettent "d'identifier et d'expliquer les inégalités persistantes ", de rendre compte du caractère intersectionnel de l'oppression et des multiples contextes dans lesquels cette oppression s'inscrit et se déploie, et de reconnaître et cultiver l'agentivité de ses victimes, c'est-à-dire leur capacité à contribuer à un changement social critique (Haslanger 2000, 36). J'illustrerai davantage la pertinence et l'utilité de l'approche améliorative en l'appliquant au concept de culture dans la troisième partie de cet article, où je me pencherai sur les conditions délibératives de la réalisation de certains droits humains, en particulier le droit des minorités culturelles de participer aux décisions nationales ou régionales les concernant.

Pour conclure cette section, si l'on reconnaît les injustices affectant les groupes sociaux minoritaires, il faut leur réserver une place propre et admettre une troisième catégorie de minorité culturelle (les minorités sociales) aux côtés des minorités nationales et ethniques. Reconnaître que les minorités sociales se retrouvent à travers les minorités nationales et ethniques montre non pas que leurs intérêts peuvent être subsumés sous ceux de leurs groupes nationaux ou ethniques respectifs, mais au contraire qu'elles demandent un traitement distinct en vertu de leur intersection avec ces autres axes d'appartenance, comme le démontrent les analyses de l'oppression et de l'intersectionnalité présentées plus haut. Alors seulement on sera en mesure de réaliser notre objectif normatif, c'est-à-dire d'envisager des solutions aux injustices sur les plans national, ethnique et social. C'est précisément en cela que consiste la formulation de droits différenciés.

\subsection{Droits différenciés}

Les droits différenciés ont en effet pour but d'assurer des protections particulières aux minorités culturelles selon les différents types d'obstacle auxquels elles font face dans leurs contextes respectifs en raison de leur statut 
minoritaire. Ainsi, différents types de droit différencié s'adresseront aux différents types de minorité. En d'autres termes, les droits différenciés auront différents objets (différents types de protection) et différents titulaires (différents types de minorité). Après avoir identifié les deux premiers types de minorité culturelle présentés ci-dessus (minorités nationales et minorités ethniques), Will Kymlicka (I995) distingue entre trois types de droit différencié pour ces groupes: le droit à l'autonomie gouvernementale, les droits polyethniques, et les droits de représentation spéciale. Le droit à l'autonomie gouvernementale (c'est-à-dire à un certain degré d'indépendance politique au sein de l'État, tel qu'illustré par le cas du fédéralisme) s'adresse spécifiquement aux minorités nationales; les droits polyethniques (tels que l'exemption pour les Sikhs de la loi obligeant les motoristes à porter un casque) s'adressent spécifiquement aux minorités ethniques; et les droits de représentation spéciale (assurant par exemple qu'un certain nombre de sièges revienne à certains groupes dans les institutions gouvernementales) s'adressent aux deux types de groupes.

Jacob Levy (2000) distingue quant à lui entre huit types de droit différencié ou de revendication de droit culturelle. Sa classification reprend les trois types d'objet sur lesquels les droits différenciés peuvent porter selon Kymlicka, et y en ajoute cinq autres. Levy identifie ainsi les types de droit différencié suivants: (i) exemptions de lois qui sinon pénaliseraient indument certaines minorités culturelles (par exemple, l'exemption du port du casque pour les Sikhs, de l'enseignement obligatoire jusqu'à dixhuit ans pour les Amish, ou de certaines régulations concernant la consommation de drogues ou la chasse pour certains groupes autochtones); (ii) assistance pour atteindre certains objectifs que la majorité peut atteindre sans assistance en vertu de son statut majoritaire - et l'on pourrait ajouter, plus précisément, privilégié - (par exemple, bulletins de vote multilingues, discrimination positive, financement d'associations culturelles minoritaires); (iii) autonomie gouvernementale pour les minorités nationales (par exemple, Québec, Catalogne ou Écosse); (iv) restrictions externes de la liberté des non-membres d'une minorité afin de protéger la culture minoritaire (par exemple, restriction de l'utilisation de l'anglais au Québec ou du vote des non-autochtones dans les territoires autochtones); (v) restrictions internes de la conduite des membres se traduisant par des formes d'ostracisme (par exemple, mise à l'écart chez les Mennonites); (vi) reconnaissance d'un code légal minoritaire par le système légal majoritaire (par exemple, droits territoriaux autochtones ou droit familial dit "traditionnel»); (vii) représentation spéciale des minorités culturelles dans les institutions gouvernementales (par exemple, réservation de certains sièges); (viii) démarches symboliques visant à reconnaître le statut valorisé des minorités culturelles (par exemple, nom de certains lieux ou groupes, jours fériés légaux, enseignement de l'histoire, excuses de la part du gouvernement). 
Bien que Levy (à la différence de Kymlicka) estime qu'il ne soit pas particulièrement utile ou pertinent de classer ces différents types de droit différencié selon les différents types de minorité culturelle auxquels ils s'adressent (2000, I26), il importe toutefois de souligner que sa classification (à la différence de celle de Kymlicka) ${ }^{3}$ fait place au troisième type de minorité présenté plus haut: les minorités sociales ${ }^{4}$. Les minorités sociales peuvent en effet faire partie des titulaires visés par les droits différenciés d'assistance (la discrimination positive pouvant s'adresser aussi bien aux minorités sociales qu'aux minorités nationales ou ethniques); par les droits de représentation spéciale (Levy cite l'exemple des circonscriptions électorales à majorité noire aux États-Unis: 2000, I27); et par les droits de démarches symboliques (enseignement de l'histoire de l'extension des libertés et droits civils aux femmes, aux pauvres et aux noirs, par exemple). On peut en outre préciser la manière dont la typologie de Levy complète celle de Kymlicka à d'autres égards encore. En effet, les démarches symboliques s'appliquent aux trois types de minorité culturelle; les restrictions externes s'appliquent aux minorités nationales (et pourraient se concevoir comme subsumées sous la catégorie d'autonomie gouvernementale puisqu'elles en sont la conséquence); et les restrictions internes ainsi que la reconnaissance de codes légaux minoritaires s'appliquent toutes deux à la fois aux minorités nationales et aux minorités ethniques, et rejoignent en ce sens les exemptions de lois.

\section{Droits différenciés et droits humains: tensions potentielles}

Nous sommes à présent en mesure de relier la question des minorités culturelles et des droits différenciés à celle des droits humains. Pour ce faire, il est utile de partir de l'observation suivante, qui semble révéler une tension entre les deux types de droit: les droits humains concernent tous les individus, tandis que les droits différenciés concernent certains groupes en particulier. Il y a là potentiellement deux points de conflit devant être résolus: l'un concernant les titulaires des droits (individus ou groupes); l'autre concernant la portée des droits (universaliste ou particulariste). Les droits humains et les droits différenciés seraient-ils donc inconciliables?

\subsection{Droits individuels et droits collectifs}

Au premier point de tension potentiel, on peut répondre plusieurs choses. Premièrement, on peut douter que la classification entre droits individuels

3. Comme mentionné plus haut, Kymlicka développe explicitement une approche qui traite principalement des droits des minorités nationales et ethniques, et non des minorités sociales (I995, I9).

4. Levy, comme Kymlicka, pense avant tout aux minorités nationales et ethniques en dressant sa classification; mais, contrairement à Kymlicka, il reconnaît que sa classification pourrait être utile aux minorités sociales en tant que type distinct (2000, I 27, note 4). 
d'une part, et droits collectifs d'autre part, soit particulièrement éclairante ou pertinente (Levy 2000, I25-I26). En effet, il semble que certains droits généralement considérés comme individuels peuvent être redéfinis comme collectifs, et inversement. Ainsi, certains auteurs présentent par exemple les protections contre la discrimination et le meurtre, dans des cas comme l'apartheid et le génocide, comme des droits collectifs plutôt qu'individuels; ou réduisent le droit collectif d'autonomie gouvernementale au droit individuel de pratiquer sa propre culture.

Deuxièmement, même en admettant la distinction largement répandue entre droits individuels et droits collectifs, il faut souligner que les droits différenciés selon l'appartenance à un groupe ne sont pas nécessairement des droits collectifs (Song 20I4). Les titulaires des droits différenciés présentés plus haut, tels que les exemptions de lois (ne pas devoir porter de casque) ou l'assistance particulière (la discrimination positive), sont des individus et non des groupes. Les individus jouissent de ce droit en vertu de leur appartenance à un certain groupe, mais le groupe lui-même n'est pas le titulaire du droit dans ces cas-là; ce sont ses membres individuels qui le sont.

Troisièmement, on peut souligner que même les droits différenciés collectifs (comme l'autonomie gouvernementale pour les minorités nationales) peuvent se justifier ultimement en vertu de l'importance que revêt le groupe pour les individus qui en font partie (Kymlicka I995; Miller I995; Tamir I993). En ce sens, le groupe a une valeur instrumentale (plutôt qu'intrinsèque): c'est l'individu, et non le groupe, qui constitue l'objet ultime de préoccupation morale. Prenons le cas de la culture nationale, formée d'une certaine langue, d'une certaine histoire, de certains emblèmes, de certaines pratiques, croyances, etc. Cette culture sous-tend et est reflétée dans les multiples institutions et sphères de la société: gouvernement, enseignement, économie, médias, sports, loisirs, etc. La culture nationale se déploie ainsi sur plusieurs plans et présente donc un aspect englobant, imprégnant souvent profondément la vie de ses membres, pour qui elle constitue un contexte particulier de sens et de valeur. On voit ainsi comment l'individu peut se retrouver au cœur de la justification des droits différenciés collectifs. Il convient par ailleurs de noter au passage que rien dans cet argument en faveur des droits différenciés n'implique une conception essentialiste, immuable, stable, ou homogène de la culture. Les cultures sont des entités sociales, dynamiques, en flux, et hétérogènes.

Quatrièmement, la protection de certains droits humains individuels énoncés dans la Déclaration universelle des droits de l'homme (tels que la liberté de religion ou la non-discrimination) passe par la protection de droits différenciés (par exemple, les exemptions de lois ou la discrimination positive). De plus, de nombreux traités de droits humains concernent spécifiquement les minorités culturelles: outre le Pacte international relatif aux droits civils et politiques et le Pacte international relatif aux droits économiques, sociaux et culturels, on peut relever la Convention internationale sur l'élimi- 
nation de toutes les formes de discrimination raciale; la Convention internationale sur l'élimination de toutes les formes de discrimination à l'égard des femmes; la Déclaration sur l'élimination de toutes formes d'intolérance et de discrimination fondées sur la religion ou la conviction; ainsi que la Déclaration des droits des personnes appartenant à des minorités nationales ou ethniques, religieuses et linguistiques. Cela veut dire que la protection des droits humains et des individus requiert également la protection des droits différenciés et des groupes auxquels ces individus appartiennent (Lukes I993). En ce sens, il y a non pas conflit mais bien complémentarité entre l'aspect individualiste des droits humains et l'aspect collectiviste des droits différenciés, la réalisation de ces derniers étant nécessaire à la réalisation des premiers.

En résumé, ce qui semble de prime abord être un conflit sur le plan conceptuel entre l'aspect individualiste des droits humains et l'aspect collectiviste des droits différenciés se révèle être un faux dilemme à la lumière de l'ontologie sociale, c'est-à-dire de la relation entre individus et groupes et, par extension, entre droits humains et droits différenciés. En effet, (i) certains droits peuvent être décrits comme ayant à la fois des titulaires individuels et des titulaires collectifs; (ii) les titulaires de certains droits différenciés sont des individus; (iii) la justification de certains droits différenciés collectifs peut être individuelle, étant donné l'importance, pour l'individu, du groupe auquel il appartient; (iv) protéger les droits humains et les individus exige de protéger les droits différenciés et les groupes dont ces individus sont membres.

Tout cela permet de résoudre le premier point de tension entre droits humains et droits différenciés, qui concernait leurs titulaires respectifs (individus ou groupes). Il se pourrait cependant que ces deux types de droit entrent en conflit au niveau de leur portée. Qu'en est-il donc du deuxième point de tension, concernant la portée respective (universaliste ou particulariste) des droits humains et des droits différenciés?

\subsection{Universalisme et particularisme}

Bien que l'on vienne de voir que de nombreux traités de droits humains concernent directement les minorités culturelles, on peut toutefois se demander s'il n'y a pas en principe une tension entre l'aspect universaliste des droits humains et l'aspect particulariste des droits différenciés. Si tous les individus ont droit à une considération égale, comment justifier le traitement spécial accordé aux minorités culturelles?

Ce deuxième point de tension potentiel peut être résolu en soulignant le contexte particulier dans lequel se trouvent les minorités culturelles par défaut, à savoir une situation d'inégalité. Dans ce contexte, l'attention particulière portée aux minorités culturelles par le biais des droits différenciés se justifie comme correcteur d'un statu quo inégalitaire où l'attention est par défaut portée à la majorité. Comme le souligne Kymlicka, les États ne sont 
pas (et ne sauraient être) neutres, que ce soit sur le plan ethnique ou national: ce sont inévitablement une certaine langue, histoire, voire religion qui se trouvent véhiculées et représentées à travers leurs différentes institutions (gouvernement, enseignement, lois, symboles, congés légaux, etc.). Traiter les minorités culturelles comme des égales par rapport au groupe majoritaire requiert de les protéger par des droits différenciés (Kymlicka 1995). De la même manière, l'existence même de groupes sociaux (genre, race, classe, orientation sexuelle, etc.) qui, comme nous l'avons vu, sont le produit de certaines relations de pouvoir sociales, signale un contexte inégalitaire dans lequel certains groupes se voient systémiquement désavantagés tandis que d'autres, inversement, s'y voient systémiquement privilégiés (Bailey I998; Frye 1983; Young 1990).

Pour défaire la tension apparente entre la notion d'égale considération requise par l'aspect universaliste des droits humains et la notion de traitement différentiel inhérente à l'aspect particulariste des droits différenciés, il est utile de distinguer entre deux types de droit différents: le droit d'être traité comme un égal et le droit à un traitement matériel égal (Dworkin I977); ainsi qu'entre deux types différents de traitement matériel inégal: un traitement différentiel et un traitement préférentiel. Le droit d'être traité comme un égal correspond au droit d'être traité avec une considération égale. Le droit à un traitement matériel égal correspond au droit à une distribution égale d'une certaine opportunité ou d'un certain bien. De ces deux types de droit, seul le droit d'être traité comme un égal est fondamental; le droit à un traitement matériel égal est un droit dérivé (Dworkin 1977). Par exemple, le droit de vote dans les démocraties libérales occidentales accorde à chaque personne un vote (et un seul) parce qu'accorder le vote uniquement à certains individus (les hommes ou les blancs ou les propriétaires) plutôt qu'à tous, ou accorder plus d'un vote à certains et pas à d'autres, reviendrait à ne pas traiter tous les individus avec une égale considération, c'est-à-dire à ne pas les traiter comme des égaux. Le droit à une part égale de l'opportunité ou du bien que constitue le vote dérive donc du droit fondamental d'être traité comme un égal.

Les individus n'ont donc un droit à un traitement matériel égal que si cela est requis par le droit fondamental d'être traité comme un égal. Ainsi, le droit d'être traité comme un égal peut parfois au contraire requérir un traitement matériel inégal (comme c'est le cas par exemple avec les exemptions de loi ou la discrimination positive). Dans ce cas, il s'agit bien d'un traitement différentiel (que je propose de définir comme un traitement matériel inégal requis par le droit fondamental d'être traité comme un égal) mais non d'un traitement préférentiel (que je propose de définir comme un traitement matériel inégal violant le droit fondamental d'être traité comme un égal). Le traitement différentiel (et non préférentiel) dont jouissent les minorités culturelles sous un régime de droits différenciés permet donc d'empêcher que la majorité ne bénéficie d'un traitement préférentiel par défaut. 
Autrement dit, les droits différenciés ne font qu'égaliser un terrain qui est sinon par défaut inégalitaire (Harris et Narayan I999; Kymlicka I995; Kymlicka 200I).

Ces deux distinctions - entre droit fondamental d'être traité comme un égal et droit dérivé à un traitement matériel égal, ainsi qu'entre traitement différentiel et traitement préférentiel - permettent de clarifier la confusion qui caractérise bien des débats. Elle permet ainsi de contrer une objection potentielle aux droits différenciés qui relève d'une telle confusion: à savoir que les droits différenciés représentent un coût indu pour la majorité (Kymlicka I995; Levy 2000). Par exemple, si à compétences égales on accorde une place à l'université à un étudiant noir ou à un étudiant pauvre, cela représenterait selon l'objection un coût pour les étudiants blancs ou les étudiants riches. Mais il est trompeur de présenter les droits différenciés en termes de coût pour la majorité, et ce, pour deux raisons.

Premièrement, parler en termes de coût revient à commettre une pétition de principe en ce que cela suppose que la priorité des blancs et des riches en matière d'admission est déjà un dû. Or c'est précisément ce point qui est en question dans la discussion de la justice sociale : qui a droit à quoi, c'est effectivement cela que la discussion vise à établir, selon des critères qui cherchent à remédier à l'injustice caractérisant le statu quo. S'il y a un coût, il tient uniquement dans le fait de voir une situation de traitement préférentiel en faveur des majorités (contraire à ce qu'exige le droit fondamental d'être traité comme un égal) se transformer en une situation de traitement différentiel pour les minorités (conforme à ce qu'exige le droit fondamental d'être traité comme un égal). Si l'on souhaite conserver la terminologie du coût pour caractériser la suppression du privilège et de la subordination, il faut alors préciser qu'il s'agit d'un coût légitime, qui n'est un coût (un retrait de bien ou d'opportunité) que parce qu'il relève d'une distribution de biens ou d'opportunités qui est injustifiée dès le départ. Pour réussir, l'objection du coût doit donc montrer non seulement qu'il y a un coût, mais que ce coût est illégitime.

Il est en outre trompeur de parler en termes de coût en ce que cela semble suggérer que la question des droits différenciés se réduit à un calcul utilitariste de coûts et de bénéfices, alors qu'il s'agit avant tout d'une question de droits. L'utilisation du critère de Pareto-optimalité (selon lequel il est impossible d'améliorer la situation d'une personne sans détériorer la situation d'une autre) n'est justifiée que si le statu quo est juste (Higgins 20I3). $\mathrm{Si}$ le statu quo est injuste, le fait que certains individus se voient retirer leur privilège afin que certains individus se voient retirer leur subordination ne représente pas une source de préoccupation morale, mais au contraire un pas vers la réalisation de la justice.

Afin de satisfaire à l'exigence améliorative introduite plus haut, il est donc crucial de ne pas se méprendre quant à l'ontologie sociale pertinente, qui met en lumière non seulement les objets ou acteurs sociaux impliqués 
(groupes minoritaires et majoritaires), mais également les processus sociaux qui les produisent. Il convient donc de ne pas aborder la question des droits différenciés d'une manière microscopique ou décontextualisée, qui éclipse les relations de pouvoir qui tissent pourtant la toile sociale inégalitaire dans laquelle les minorités se situent. Envisagée sous l'angle amélioratif, la tension supposée entre l'universalisme des droits humains et le particularisme des droits différenciés disparaît. L'angle amélioratif permet en effet de voir en quoi la visée particulariste des droits différenciés, loin de s'y opposer, est en réalité nécessaire pour réaliser la visée universaliste des droits humains; c'est-à-dire de voir en quoi la réalisation des droits humains requiert la mise en place de droits différenciés.

\section{Minorités culturelles et droits humains: pour une délibération inclusive}

La pertinence de l'approche améliorative se révèle encore si l'on se penche sur les nombreux débats qu'ont suscités les démarches de législation concernant le port du voile ou du niqab dans les démocraties libérales occidentales au cours des dix dernières années, que ce soit en France, en Belgique, aux Pays-Bas ou au Québec. Dans cette dernière section, je prends comme points de référence les droits humains, énoncés dans la Déclaration des droits des personnes appartenant à des minorités nationales ou ethniques, religieuses et linguistiques, «de jouir de leur propre culture, de professer et de pratiquer leur propre religion et d'utiliser leur propre langue, en privé et en public, librement et sans ingérence ni discrimination quelconque » (article 2.I); «de participer pleinement à la vie culturelle, religieuse, sociale, économique et publique " (article 2.2); et "de prendre une part effective, au niveau national et, le cas échéant, au niveau régional, aux décisions qui concernent la minorité à laquelle elles appartiennent ou les régions dans lesquelles elles vivent, selon des modalités qui ne soient pas incompatibles avec la législation nationale» (article 2.3). Je me penche ici en particulier sur la situation des minorités musulmanes dans les démocraties libérales occidentales pour dégager les conditions délibératives de la réalisation de ces droits humains. Ces droits humains étant posés et adoptés comme objectifs, il convient en effet de délibérer sur la manière dont ils peuvent être réalisés. Or cette délibération n'a de sens que si elle inclut toutes les parties concernées.

Il faut commencer par noter que les démarches de législation concernant le port du voile ou du niqab (qu'elles aillent dans le sens de son interdiction à l'école, dans la rue, ou dans la fonction publique) vont clairement à l'encontre des droits énoncés à l'article 2, alinéas I et 2, puisqu'elles limitent la pratique de la religion en public, imposant ainsi un choix entre la pleine participation à la vie culturelle et religieuse et la pleine participation à la vie sociale, économique et publique. Je ne souhaite pas ici évaluer dans quelle mesure la laïcité ou le sécularisme peut ou non justifier de telles démarches (Brown 20I3; Spitz 20I4). Je souhaite plutôt m'attarder sur les exigences délibératives requises pour réaliser le but énoncé à l'article 2, alinéa 3, à 
savoir la participation des minorités aux décisions nationales ou régionales les concernant. L'urgence de cette question s'impose en effet étant donné l'exclusion délibérative prépondérante des femmes et des communautés musulmanes des débats les concernant.

Afin de pouvoir dégager les conditions délibératives de la réalisation des droits humains qui nous préoccupent ici, j'adopte l'approche améliorative. Celle-ci permet de voir en quoi les processus à l'œuvre historiquement dans le colonialisme territorial à travers le monde se retrouvent aujourd'hui dans ce que l'on pourrait appeler la colonisation discursive au sein des sociétés multiculturelles; et en quoi ces processus impliquent et affectent le concept de culture, qui vient stigmatiser les uns et auto-légitimer les autres. Le concept de culture joue ainsi un rôle problématique qu'il importe d'identifier dès lors que nous cherchons à réaliser nos objectifs de justice, en l'occurrence une délibération inclusive pour la réalisation des droits humains susmentionnés.

Le souci de «civiliser » des cultures jugées par l'Occident comme problématiquement traditionnelles n'est pas neuf. Pourtant, l'histoire du colonialisme révèle que les "traditions" mêmes censées justifier en partie l'entreprise coloniale sont en fait souvent le produit des réactions au contact avec les colons (Jaggar 2005). Ainsi, la recrudescence de la pratique de la clitoridectomie fit partie de l'opposition des nationalistes kenyans aux colons britanniques (Pollitt I999); et celle de la pratique du sati en Inde fut le résultat du débat qui tenta de la présenter comme un symbole de la culture hindoue et indienne (Narayan I 997). Ces pratiques «traditionnelles » vinrent alors renforcer la légitimité du projet colonial.

De la même manière, dans le contexte des débats suscités par les démarches de législation concernant le port du voile ou du niqab, l'exclusion délibérative des minorités en question est d'autant plus problématique qu'elle peut provoquer leur radicalisation suite à des réactions d'affirmation «culturelle» de plus en plus conservatrices en vue de protéger leur identité (An-Na'im 2003). Cela a pour effet, comme dans le cas du colonialisme, de transformer les accusations extérieures a priori non fondées de conservatisme en une sorte de prophétie auto-réalisatrice - effaçant ainsi le rôle causal fondamental que l'exclusion de la minorité par la majorité joue dans ce processus de radicalisation. Comme dans le cas du colonialisme, la culture minoritaire se voit ensuite présentée non seulement comme naturellement ou essentiellement conservatrice ou oppressive, mais également comme une entité hermétique évoluant indépendamment du reste de la société, comme si elle était entièrement déconnectée des rapports de force extérieurs pourtant si causalement puissants à son égard. Ici, les limites des approches purement conceptuelle ou purement descriptive apparaissent clairement. Adopter la conception ordinaire - ici, naturalisante ou essentialisante - du concept de culture, constituera un véritable obstacle épistémologique à une description correcte, sur le plan ontolo- 
gique, des mécanismes et des rapports de force socio-politiques qui la soustendent et la produisent.

On voit ici toute l'importance d'adopter une approche améliorative, qui permet de voir que la culture (à l'instar de la catégorie femmes évoquée plus haut par exemple) est elle-même le produit de relations de pouvoir politiques et sociales. Comme on l'a vu, l'approche améliorative ne cherche pas simplement à expliquer la compréhension ordinaire d'un certain concept (comme l'approche conceptuelle), ni simplement à identifier son extension empirique (comme l'approche descriptive). L'approche améliorative vise à formuler un concept (ici, celui de culture) qui soit utile pour atteindre nos buts légitimes de moralité politique (ici, sortir d'un statu quo qui s'autolégitimise en stigmatisant certains groupes ou cultures). Cette approche permet donc d'identifier l'ontologie sociale pertinente, c'est-à-dire les acteurs (groupes ou cultures) minoritaire et majoritaire impliqués, ainsi que les relations de pouvoir dont ils résultent et qui structurent leurs interactions. On dispose alors des ressources analytiques nécessaires à la fois pour saisir et pour défaire ces relations de pouvoir, ainsi que les injustices d'exclusion et de subordination qu'elles engendrent.

Même en l'absence de ce processus de radicalisation, l'exclusion des minorités des débats les concernant résulte en une construction unilatérale d'un certain discours à leur sujet: certaines cultures comme étant naturellement oppressives et inégalitaires; certains de leurs membres comme étant essentiellement opprimés et subordonnés (Al-Faruqi I999; Al-Saji 2008; Keck et Sikkink I998; Mohanty 2003; Narayan 1997). Ce monologue se traduit par une colonisation discursive, qui impose des catégories d'analyse monolithiques et anhistoriques de "la» femme et de "la» culture musulmanes (Mohanty 2003). Ontologiquement, cela suppose que la catégorie «femme musulmane» est une réalité fixée d'avance, qui préexiste à ses membres individuels et qui, par définition, leur retire toute agentivité (Mohanty 2003), y compris leur agentivité délibérative. Ce discours vient alors renforcer à la fois la légitimité des démarches de législation à leur égard et la légitimité de l'exclusion délibérative qui rend ces démarches possibles.

Pour pouvoir sortir de cette impasse délibérative et ainsi garantir la participation des minorités aux décisions nationales ou régionales les concernant (conformément aux droits humains énoncés dans la Déclaration des droits des personnes appartenant à des minorités nationales ou ethniques, religieuses et linguistiques), il faut transférer le fardeau de la preuve: c'est-à-dire interroger les pratiques, croyances et institutions non seulement des minorités, mais également de la majorité - et en particulier le rôle que cette dernière joue dans les débats et réactions sociales auxquels on assiste. Autrement dit, il faut rendre compte des processus sociaux et des rapports de force qui sous-tendent la situation qui nous préoccupe. Il faut révéler la responsabilité causale de la majorité dans le discours public (son monologue "délibératif» résultant en une forme de colonisation discursive 
venant légitimer les démarches de législation) et reconnaître l'agentivité délibérative des minorités et de leurs membres en les incluant dans les délibéra-

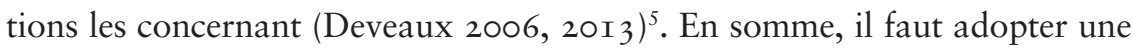
approche améliorative. Celle-ci nous fournit en effet les outils conceptuels et les catégories d'analyse qui nous permettent "d'identifier et d'expliquer les inégalités persistantes"; de rendre compte du caractère intersectionnel de l'oppression et des multiples contextes dans lesquels cette oppression s'inscrit et se déploie; et de reconnaître et cultiver l'agentivité de ses victimes, c'est-àdire leur capacité à contribuer à un changement social critique (Haslanger $2000,36)$. L'approche améliorative permet ainsi de voir comment la réalisation des droits humains peut effectivement protéger les minorités culturelles.

\section{Conclusion}

Dans cet article, j'ai identifié et interrogé les tensions potentielles entre minorités culturelles et droits humains, afin de montrer en quoi les droits humains peuvent protéger les minorités culturelles et, ultimement, de problématiser la manière dont cette protection peut se déployer. Après avoir écarté le défi relativiste, j'ai montré en quoi les aspects collectiviste et particulariste des droits différenciés ne se trouvent pas en tension respectivement avec les aspects individualiste et universaliste des droits humains. À partir de certains droits humains, j'ai alors examiné la situation des minorités musulmanes dans les démocraties libérales occidentales pour dégager les conditions délibératives de la réalisation de ces droits humains. Tout au long de mon analyse, j'ai mis en lumière les enjeux méthodologiques qui soustendent ces différents débats et montré la nécessité d'adopter une approche méthodologique améliorative pour réaliser nos objectifs de justice.

\section{Bibliographie}

Ake, Claude. 1987. "The African Context of Human Rights", Africa Today 34: I-2, 5-I3.

Al-Faruqi, Maysam. 1999. "Women's Self-Identity in the Qur'an and Islamic Law”, in Gisela Webb (ed.), Windows of Faith: Muslim Women's Scholarship Activism in the United States, Syracuse, NY, Syracuse University Press, 72-Ior.

Al-Saji, Alia. 2008. "Voiles racialisés ", Les Ateliers de l'Éthique 3: 2, 39-55.

An-Na'im, Abdullahi. 2003. "Introduction: 'Area Expressions' and the Universality of Human Rights: Mediating a Contingent Relationship", in David P. Forsythe and Patrice C. MacMahon (eds.) Human Rights and Diversity: Area Studies Revisited, Lincoln, NE, University of Nebraska Press, I-2I.

5. Je ne peux, dans le cadre et l'espace de cet article, m'attarder davantage ni sur les conditions nécessaires à une délibération réellement (et non simplement formellement) inclusive, ni sur la justification normative de ces conditions. Pour un traitement plus approfondi de ces conditions délibératives et de leur justification normative, voir Catala 20 I 5 , où je soutiens qu'une délibération réellement inclusive et juste requiert la justice et la confiance épistémiques, conditions qui sont non seulement justifiées mais exigées par trois engagements fondamentaux de la démocratie délibérative, à savoir l'égalité, la légitimité et l'imputabilité. 
Bailey, Alison. I998. "Privilege”, Journal of Social Philosophy 29: 3, I04-I I9.

Bauer, Joanne et Daniel Bell (eds.). I999. The East Asian Challenge for Human Rights, New York, Cambridge University Press.

Bayefsky, Anne. 200I. The UN Human Rights Treaty System: Universality at the Crossroads, Ardsley, NY: Transnational Publishers.

Beitz, Charles. 2009. The Idea of Human Rights, Oxford, Oxford University Press.

Brown, Wendy. 20I3. "Civilizational Delusions: Secularism, Tolerance, Equality", in Maillé et al. (eds.), Revealing Democracy: Secularism and Religion in Liberal Democratic States, Bruxelles, Peter Lang, 35-56.

Catala, Amandine. 20I 5. "Democracy, Trust, and Epistemic Justice", The Monist 98: 4, 424-440.

Crenshaw, Kimberle. I99I. "Mapping the Margins: Intersectionality, Identity Politics, and Violence against Women of Color", Stanford Law Review 43: 6, I 24 I-I 299.

Deveaux, Monique. 2006. Gender and Justice in Multicultural Liberal States, New York, Oxford University Press.

Deveaux, Monique. 2013. "Regimes of Accommodation, Hierarchies of Rights", in Maillé et al. (eds.), Revealing democracy: Secularism and Religion in Liberal Democratic States, Bruxelles, Peter Lang, 77-93.

Dworkin, Ronald. 1977. Taking Rights Seriously, Harvard University Press.

Frye, Marilyn. 1983. The Politics of Reality: Essays in Feminist Theory, Crossing Press.

Harris, Luke Charles and Uma Narayan, I999. "Affirmative Action as Equalizing Opportunity: Challenging the Myth of 'Preferential Treatment' ", National Black Law Journal I 6: 2, I27-I43.

Haslanger, Sally. 2000. "Gender and Race: (What) Are They? (What) Do We Want Them to Be? ", Noûs 34: I, 3I-55.

- 2005. "What Are We Talking About? The Semantics and Politics of Social Kinds", Hypatia 20: 4, I0-26.

Higgins, Peter. 2013. Immigration Justice, Edinburgh, Edinburgh University Press.

Jaggar, Alison. 2005. “' 'Saving Amina': Global Justice for Women and Intercultural Dialogue ", Ethics \& International Affairs 19: 3, 55-75.

Keck, Margaret and Kathryn Sikkink. I998. Activists Beyond Borders: Advocacy Networks in International Politics, Ithaca, NY, Cornell University Press.

Kymlicka, Will. I995. Multicultural Citizenship: A Liberal Theory of Minority Rights, New York, Oxford University Press.

- 200I. Politics in the Vernacular: Nationalism, Multiculturalism, and Citizenship, New York, Oxford University Press.

Levy, Jacob. 2000. The Multiculturalism of Fear, New York, Oxford University Press.

Lukes, Steven. I993. "Five Fables About Human Rights", in S. Shute and S. Hurley (eds.), On Human Rights: The Oxford Amnesty Lectures, New York, Basic Books, 19-40.

Miller, David. I995. On Nationality, New York, Oxford University Press.

Mills, Charles. 2005. "Ideal Theory as Ideology", Hypatia 20: 3, I65-183.

Mohanty, Chandra Talpade. 2003. Feminism without Borders: Decolonizing Theory, Practicing Solidarity, Duke University Press. 
Narayan, Uma. I997. Dislocating Cultures: Identities, Traditions, and Third World Feminism, New York, Routledge.

Nickel, James. 20I4. "Human Rights", The Stanford Encyclopedia of Philosophy (Spring 20I4 Edition), Edward N. Zalta (ed.):

[http://plato.stanford.edu/archives/spr2oI4/entries/rights-human/].

Nickel, James and David Reidy. 2008. "Relativism, Self-Determination, and Human Rights", in Deen Chaterjee (ed.), Democracy in a Global World: Human Rights and Participation in the 21st Century, Lanham, MD, Rowman \& Littlefield.

Organisation des Nations Unies. I948. Déclaration universelle des droits de l'homme [http://www.ohchr.org/EN/UDHR/Pages/Language.aspx ? LangID=frn].

—. I965. Convention internationale sur l'élimination de toutes les formes de discrimination raciale

[http://www.ohchr.org/FR/ProfessionalInterest/Pages/CERD.aspx].

- I966. Pacte international relatif aux droits civils et politiques [http://www. ohchr.org/fr/professionalinterest/pages/ccpr.aspx].

- I966. Pacte international relatif aux droits économiques, sociaux et culturels, [http://www.ohchr.org/FR/ProfessionalInterest/Pages/CESCR.aspx].

- I979. Convention sur l'élimination de toutes les formes de discrimination à l'égard des femmes [http://www.ohchr.org/FR/ProfessionalInterest/Pages/ CEDAW.aspx].

- I98I. Déclaration sur l'élimination de toutes formes d'intolérance et de discrimination fondées sur la religion ou la conviction

[http://www.ohchr.org/FR/ProfessionalInterest/Pages/ReligionOrBelief.aspx].

- I992. Déclaration des droits des personnes appartenant à des minorités nationales ou ethniques, religieuses et linguistiques

[http://www.ohchr.org/FR/ProfessionalInterest/Pages/Minorities.aspx].

Pollitt, Katha. I999. "Whose Culture?", in Susan Okin, Is Multiculturalism Bad for Women? Princeton, NJ, Princeton University Press.

Song, Sarah. 20I4. "Multiculturalism", The Stanford Encyclopedia of Philosophy (Spring 20I4 Edition), Edward N. Zalta (ed.):

[http://plato.stanford.edu/archives/spr2or/4/entries/multiculturalism/].

Spitz, Jean-Fabien. 20I4. "La République française et le multiculturalisme", in B. Gagnon et J. Steele (dir.), Concilier démocratie et diversité: Essais de théorie politique, Bruxelles, Peter Lang, 67-96.

Talbott, William. 2005. Which Rights Should be Universal? Oxford, Oxford University Press.

Tamir, Yael. I993. Liberal Nationalism, Princeton, NJ, Princeton University Press.

Taylor, Charles. I999. "Conditions of an Unforced Consensus on Human Rights", in Joanne Bauer et Daniel Bell (eds.), The East Asian Challenge for Human Rights, New York, Cambridge University Press, I24-I44.

Young, Iris Marion. I990. Justice and the Politics of Difference, Princeton, NJ, Princeton University Press. 